\title{
THE HIPPARCOS EXTRAGALACTIC LINK
}

\author{
JEAN KOVALEVSKY \\ Observatoire de la Côte d'Azur/CERGA, \\ Av. Copernic 06130 Grasse, France
}

\begin{abstract}
In conformity with the IAU resolutions on reference frames adopted in 1991, the Hipparcos catalogue will represent, in the visible spectrum, the celestial reference system defined by fixed positions of extragalactic radio-sources. This will be realized by the strongest possible link between the IERS celestial reference frame with positions and/or proper motions of the largest possible number of Hipparcos stars determined also with respect to extragalactic objects. The data which will be used must be available before April 1995. It will include the following: positions and proper motions of radio stars observed by VLBI, VLA and MERLIN; photographic positions in fields including quasars; proper motions with respect to galaxies of the Lick, Yale, and Kiev programs, proper motions derived from pairs of photographic plates taken at large time intervals; and possibly data acquired by Hubble Space Telescope and from Earth's rotation data. The organization of the tasks within the working group is briefly described. The final accuracy of the link is expected to be of the order of, or better than, half a milliarcsecond.
\end{abstract}

\section{Introductory remarks}

The set of recommendations that constitute the IAU resolution A4 (Bergeron, 1992) on celestial reference systems and frames states that the new space coordinate grids will have no global rotation with respect to a set of distant extragalactic objects. The conventional celestial reference frame which will represent this system will consist of positions of about 600 extragalactic radio sources the list of which is being presented at the present IAU General Assembly by the working group set up to this effect and chaired by C. de Vegt (see resolution $\mathrm{N}^{\circ} \mathrm{B} 6$, this volume). A preliminary realization of 
this frame already exists: it has been prepared by IERS. However, note 5 to recommendation VII states that as long as the relationship between the optical and the extragalactic radio frames is not sufficiently accurately determined, the FK5 catalogue shall be considered as a provisional realization of the celestial reference system in optical wavelengths.

Now that it is assessed that the Hipparcos catalogue will be considerably more precise and denser than the FK5, and that it will be in the future years the main accurate astrometric reference catalogue, it was resolved by the Hipparcos Science Team that this catalogue should be linked as tightly as possible to the fundamental reference frame in radio wavelengths so that it satisfies the demands of the IAU resolutions and hence becomes the realization of the new celestial reference system in optical wavelengths.

This task is undertaken by a working group of the Hipparcos Science Team consisting of P.D. Hemenway, K.J. Johnston, J. Kovalevsky, J.L. Lestrade, L. Lindegren, R.A. Preston, W.F. Van Altena and C. de Vegt.

\section{Method}

The principle of the link was described by Froeschlé and Kovalevsky (1982). Let us call $\mathbf{X}_{\mathbf{V}}$ and $\mathbf{X}_{\mathbf{H}}$ respectively the unit vectors of the direction of a star as observed in the extragalactic and in the Hipparcos reference frames. Similarly, $\mathbf{X}_{\mathbf{V}}{ }^{\prime}$ and $\mathbf{X}_{\mathbf{H}}{ }^{\prime}$ are the proper motions of a star in these systems. One has

$$
\begin{gathered}
\mathbf{X}_{\mathbf{H}}=R \mathbf{X}_{\mathbf{V}} \\
\mathbf{X}_{\mathbf{H}}{ }^{\prime}=R \mathbf{X}_{\mathbf{V}}^{\prime}+R^{\prime} \mathbf{X}_{\mathbf{V}}
\end{gathered}
$$

From both indices, one has

$$
\mathbf{X}\left\{\begin{array} { l } 
{ \operatorname { c o s } \delta \operatorname { c o s } \alpha , } \\
{ \operatorname { c o s } \delta \operatorname { s i n } \alpha , } \\
{ \operatorname { s i n } \delta , }
\end{array} \quad \mathbf { X } ^ { \prime } \left\{\begin{array}{c}
-\mu_{\alpha} \cos \delta \sin \alpha-\mu_{\delta} \sin \delta \cos \alpha \\
\mu_{\alpha} \cos \delta \sin \alpha-\mu_{\delta} \sin \delta \sin \alpha \\
\mu_{\alpha} \cos \delta
\end{array}\right.\right.
$$

where the rotation matrices are

$$
R=\left|\begin{array}{ccc}
1 & \gamma & -\beta \\
-\gamma & 1 & \alpha \\
\beta & -\alpha & 1
\end{array}\right| \quad \mathrm{R}^{\prime}=\left|\begin{array}{ccc}
0 & \gamma^{\prime} & -\beta^{\prime} \\
-\gamma^{\prime} & 0 & \alpha^{\prime} \\
\beta^{\prime} & -\alpha^{\prime} & 0
\end{array}\right|
$$

The work consists in writing as many observation equations (1) and (2) as possible and then solving them for $R$ and $R^{\prime}$. Then, applying the rotations $R$ 
and $R^{\prime}$ to all positions and proper motions of the Hipparcos catalogue, one will obtain a new version of the catalogue which will be in the extragalactic reference system with an accuracy to be derived from the accuracy of the conventional extragalactic reference frame used and the variance-covariance matrix of the determination of the matrices $R$ and $R^{\prime}$.

\section{Data available}

In order to determine $R$, it is necessary that the positions of the Hipparcos stars be directly determined with respect to the extragalactic radio sources belonging to the fundamental list of objects. The most precise source of such data is VLBI astrometry. A total of 14 stars are expected to be observed by the Deep Space Network and the Australian VLBI system. A sub-milliarcsecond precision is expected. However, some of these stars are double and the necessary corrections for orbital motion to represent the motion of the radio source may not be so accurately determinable. Less precise, but more numerous, are the positions of stars determined by VLA or Merlin interferometers. About 40 star positions have been obtained since 1984 with a precision of the order of 30 mas (see for instance, Florkowski et al., 1985). Some of them will be reobserved and will provide proper motions.

Another source of information are photographic plates in which positions of Hipparcos stars are determined with respect to quasars whose positions are known in the extragalactic reference frame. Some 300 such plates are available and are being measured and reduced by C. de Vegt. Although the precision of each plate measurement does not permit to determine star positions to better than $0^{\prime \prime} .1$, an accuracy of 3 mas is expected for the link using these data alone.

The most important quantity requested from the link is $R^{\prime}$. Although the best possible coincidence of the origins of the coordinate systems at epoch is very desirable, it is even more important for all kinematic and dynamical studies which will be performed with the catalogue, that the residual rotation of the coordinates be reduced to a minimum. It is to be remarked that it is not necessary that the proper motions be referred to extragalactic objects of known positions. It is sufficient that the proper motions of stars are determined with respect to extragalactic objects. So several types of data are available.

The VLBI observations provide absolute proper motions with a submilliarcsecond per year precision for the 14 stars already referred to. Reobservation by VLA and Merlin of some 10 other radio sources will provide also accurate proper motions.

Pairs of photographic plates of the same region separated by a large time interval and reduced with respect to quasars assumed to be fixed, will 
provide absolute proper motions. This will be the case for about 50 pairs of Schmidt plates taken in Tautenburg at about 25 year intervals, while a dozen of pairs of plates separated by intervals of time as long as 90 years are available in Bonn Observatory. It is to be noted that a discussion of residuals of the de Vegt quasar photography program as a function of the date may give some information on the time-dependent rotation.

A very important contribution to the link are the results of large surveys whose main objective was to measure absolute stellar proper motions with respect to an extragalactic reference. Although they were generally designed for faint stars of astrophysical interest, there is also a large number of brighter stars which are in the Hipparcos catalogue. The most important is the Lick Northern Proper Motion Program (Klemola et al., 1987). Among the 300000 measured stars, about 7000 are in the Hipparcos catalogue. A smaller program, the Yale Southern Proper Motion Program (van Altena et al., 1986), has the advantage of concentrated observations around the Southern pole. It has some 1200 stars in common with Hipparcos. Finally, the Kiev survey for the northern hemisphere has about 2500 common stars with Hipparcos.

There are also some hope that despite a late start in the program, some radial components of proper motions from quasars can be determined from Hubble Space Telescope observations. If they are not ready in time, they will provide an a-posteriori check of the link.

Another type of link was proposed by Vondrak during this general assembly of the IAU. Earth orientation parameters obtained by optical methods (astrolabes and transit instruments) during the last years will be rereduced using positions and proper motions of the Hipparcos catalogue and then compared with those deduced VLBI observations. The differences will be interpreted as a rotation and a time dependent rotation of the Hipparcos reference system in the VLBI extragalactic reference system.

\section{Status of the work}

For all the data described above, reduction software is written by the teams responsible for the various observation techniques (radio, photographic plates, surveys). A provisional catalogue of reference radio sources had been provided by IERS. The positions and proper motions from the Hipparcos 30 month combined solution were provided to all the teams. Except in the case of radio observations, only single stars will be included in the test solution which should be examined and discussed in early 1995 . There are two reasons for this decision. While the position of the radio emission in a couple is well identified within a couple of mas, the point representative of a close binary may not be the same in Hipparcos and on 
a photographic plate. In addition, the proper motion determined in a short time interval may not be well corrected for orbital motion in the present version of the catalogue.

The final version of the combined Hipparcos catalogue will be available in April 1995, so that the final link should be ready by mid-1995. At present, it is not possible to assess what will be the accuracy of the link. From the very partial results obtained and from the accuracy evaluations of various techniques (see table 1), one should guess that half a milliarcsecond in the components of both matrices R and R' could be achieved. An optimistic view is that the accuracy could be even significantly better for $R$ '.

TABLE 1. Link of Hipparcos catalogue to the extragalactic reference frame - Preliminary expectations

\begin{tabular}{lcc}
\hline Technique & $\sigma R$ (mas) & $\sigma R^{\prime}(\mathrm{mas} / \mathrm{yr})$ \\
\hline VLBI & 0.5 & 0.5 \\
VLA/MERLIN & 3 & 1 \\
HST & & 1.5 \\
QSO + Photo & 3 & $?$ \\
Photo Bonn & & 0.7 \\
Photo Potsdam & & 1 \\
Survey & & 0.3 \\
Earth's rotation & $?$ & $?$ \\
\hline
\end{tabular}

Adopting this optimistic approach and assuming an error of the link of the order of 0.25 mas per year, one can compare the evolution of the error with time as compared with the error evolution of the major catalogues and of the random errors of Hipparcos (Fig. 1). One can see from this figure how much better the Hipparcos frame will be in comparison with the Hipparcos catalogue. A major task for future astrometry will be to extract from the Hipparcos catalogue a number of stars whose positions and proper motions will be improved in order to materialize as accurately as possible this frame.

\section{Acknowledgements}

Fifteen different institutes are contributing to this very diversified task. These are, first of all, ESA, European Space Agency, responsible of Hipparcos programme and, in alphabetic order : Astronomical Institute (Czeck Republic), CSIRO/ATNF (Australia), Hamburg Observatory (Germany), Haystack Observatory (USA), JPL (USA), Kiev Observatory (Ukraine), 


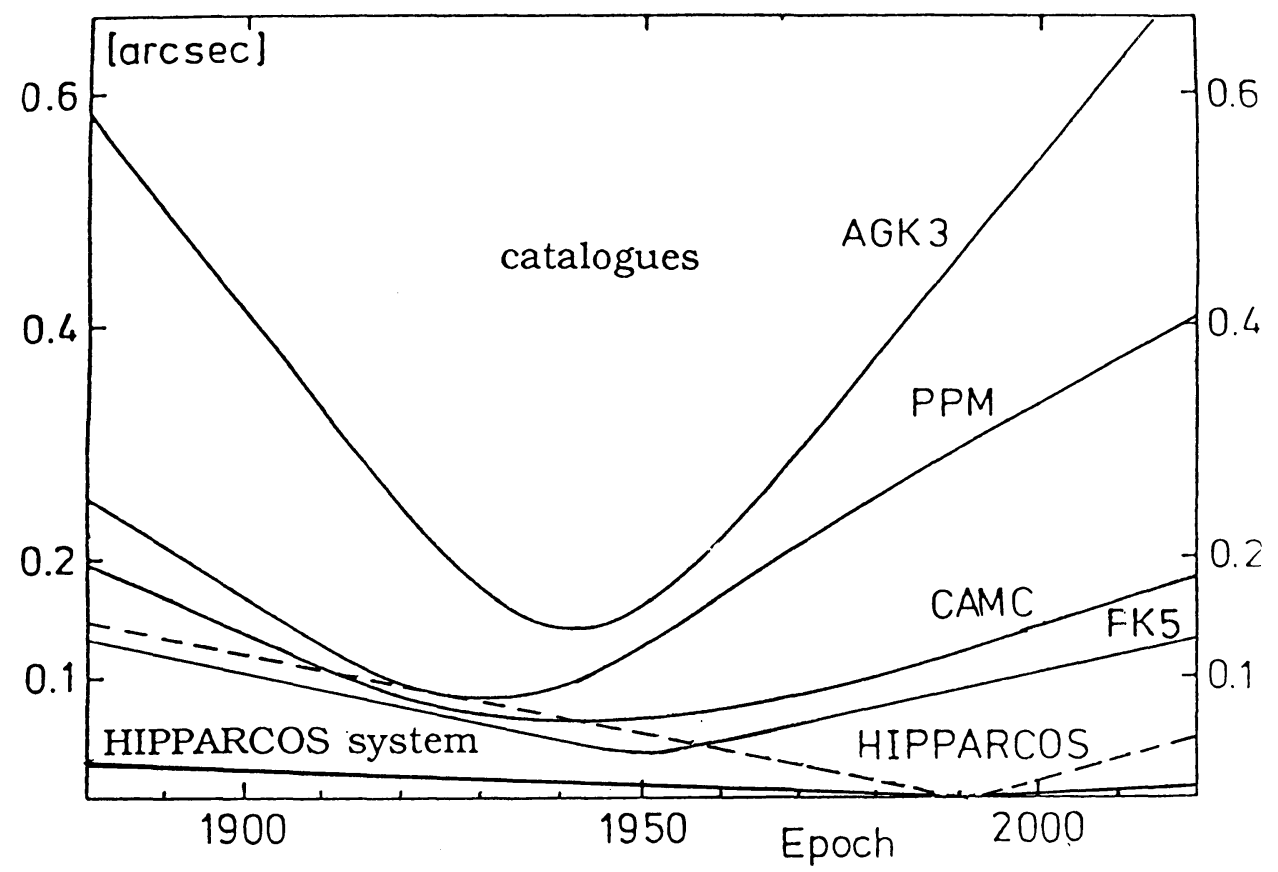

Figure 1. Evolution of the precision of various catalogues and of the Hipparcos system as a function of time

Lund Observatory (Sweden), Observatoire de la Côte d'Azur/CERGA (France), Observatoire de Paris (France), Observatorium Hoher List (Germany), Potsdam Observatory (Germany), US Naval Observatory (USA), University of Texas (USA), Yale Observatory (USA). Their contribution is greatfully acknowledged.

\section{References}

Bergeron, J. (ed.), 1992, Proceedings of the twenty-first General Assembly of the IAU, Kluwer Acad. Publ., pp 41-64.

Florkowski, D.R., Johnston, K.J., Wade, C.M. and de Vegt, C., 1985, Astron. J., 90, pp 2381-2386.

Froeschlé, M. and Kovalevsky, J., 1982, A \& A, 116, pp 89-96.

Klemola, A.R., Jones, B.F. and Hanson, R.B., Astron. J., 94, pp 501-515.

van Altena, W.F., Girard, T., Lòpez, C.E., Klemola, A.R., Jones, B.F. and Hanson, R.B., 1986, Highlights of Astronomy, J.P. Swings (ed.), 7, pp 89-92. 